\title{
A modified closed-open approach as part of a graduated and integrative approach to rhinoplasty
}

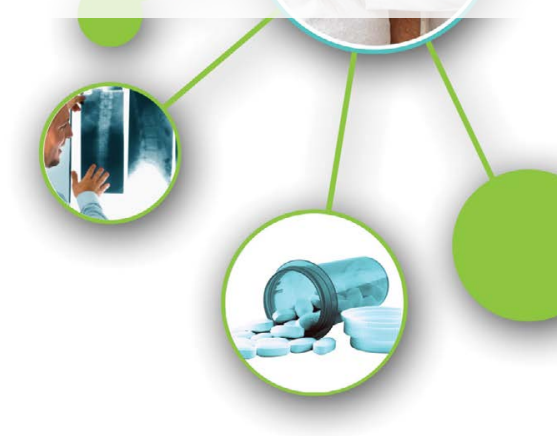

Open versus closed approach in rhinoplasty is a frequently debated topic in aesthetic plastic surgery. Although good results can often be achieved with either technique, both have unique advantages and disadvantages. In this investigation we present our experiences of a modified closed-open approach which has been applied on 482 complex primary and secondary rhinoplasties. Three representative cases are described in more detail.

Surgical method: The procedure begins as a closed approach through a intracartilaginous incision allowing cephalic trimming of the lateral crura, dorsal rasping and/or excision. Patients requiring extensive nasal tip maneuvers are subjected to exposure of the alar cartilage framework through a transcolumellar/limited marginal incision. This provides not only adequate exposure of the alar cartilages but also easy access to the septum. In our hands this approach is easy and expeditious. It requires less tip dissection, and therefore may avoid the prolonged postoperative edema which is often a consequence of open or extended closed tip delivery approaches.

\section{KEYWORDS: rhinoplasty, open \& closed approach, modified closed-open approach}

\section{Introduction}

Rhinoplasty is one of the most common surgical procedures in aesthetic plastic surgery. There has been a vivid debate over the last decades whether the open or closed approach should be used for optimal results [1,2]. Various open and closed techniques, have been described all having the goal to obtain reliable and long-term results with as little surgery as possible [3]. Less surgery causes less soft tissue trauma, ecchymosis and postoperative swelling which, taken together may shorten the recovery period [4]. However, limited surgery may also hamper the exposure, cause technical problems and undesirable results.

This article describes a modified closed-open approach. In our opinion the best candidates for this approach are selected primary or secondary cases who would benefit from the limited dissection of the closed approach but require the better exposure offered by the open approach.

This article does not contain any studies with human or animal subjects.

Informed consent was obtained from all individual participants included in the study.

\section{- Indications}

We have applied the modified closedopen technique on cases requiring substantial tip modification as the closed approach alone does not appear to be conducive to the whole gamut of tip-plasty maneuvers, such as shaping and positioning of the tip with suture techniques, placement of precise tip grafts, septal extension grafts and columellar struts. In our opinion the modified closed-open approach is preferential in such cases as it avoids unnecessary skeletonization, and yet allows undistorted visualization of the structures to be manipulated. Severe cases of deviated septum, calling for extensive scoring and inferior septal wedge excision are however best served through an approach providing wide exposure.

\section{Surgical technique}

\section{Closed approach:}

All patients are operated under general anesthesia. The surgical procedure begins by making an intracartilaginous incision as shown in FIGURE 1. Care is taken not to continue the incision too far medially leaving $4-5 \mathrm{~mm}$ of intact mucosa between the medial end of the intracartilaginous incision and the septal mucosa. This leaves the internal valve area undisturbed. Through this access, cephalic trimming is performed as necessary followed by
Jonas Röjdmark* \&

Agko Mouchammed

Akademikliniken, Department Plastic Surgery, Storängsvägen 10, Sweden

*Author for correspondence:

jonas.rojdmark@ak.se 

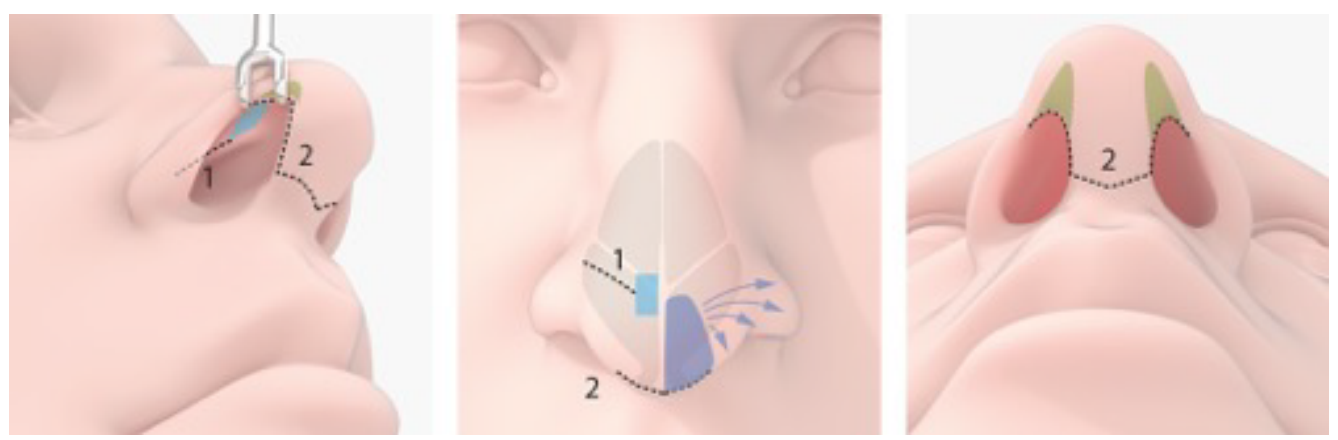

FIGURE 1 Left: The position and extent of the intracartilaginous and transcolumellar/limited marginal incisions are depicted. The alar margin is retracted to highlight that the internal valve (blue strip) is not violated by the intracartilaginous incision. The marginal incision is limited to just past the soft triangle (green). Center: Again, the intact internal nasal valve (light blue) is shown. The intact middle and lateral alar rim allows for extended drainage (arrows) of the tip (dark blue). Right: Basilar view demonstrating the position and extent of the transcolumellar/limited marginal incision in relation to the soft triangle (green).

maneuvers addressing the nasal dorsum. With the overlying skin and soft tissue envelope in anatomic position progressive sculpting of the nose can easily be performed. There is no need for dissection more than the area to be surgically modified. Continuous visual assessment of the changing aesthetics of the nose is possible without having to redrape the skin over the nose each time.

\section{Open approach:}

The surgery continues with nasal tip exposure. As illustrated in FIGURE 1, a transcolumellar incision is continued as a limited marginal incision just past the soft triangle. This leaves most of the alar rim uninterrupted and secures a broad base for the venous and lymphatic drainage of nasal tip skin sleeve, especially towards the lateral aspect, where the principal venous and lymphatic drainage is found [5] Preservation of this vascular and lymphatic connection is paramount. It is this critical aspect of the technique that differentiates it from the traditional open approach and in our belief affords less nasal tip swelling. A number of ligamentous connections in the cranial part of the nasal tip are also preserved maintaining some tip support. Through this conservative exposure technique, tip modification maneuvers - sutures or grafts - can be performed expeditiously with the cartilages in resting anatomic position as in the open approach. This is in contrast to the distortion caused by alternative extended closed approaches with tip delivery. Moreover, most septal deformities can be addressed under direct vision without having to resort to transfixion incisions, thus preserving a fully intact septal mucoperichondrium and mucosa. If indicated, osteotomies can be performed and dorsum adjustments made at any time during the procedure.

\section{- Clinical cases}

\section{Case 1}

A 24-year old woman, who previously had had open rhinoplasty, presented with an overresected nasal dorsum with visible contour irregularities, a wide nasal base and an overrotated nasal tip (labiocolumellar angle $=115$ degrees). External valve collapse and complex intranasal scarring caused breathing problems. Diced conchal cartilage wrapped in deep temporalis fascia was introduced through the endonasal approach after limited undermining of the nasal dorsum. Lateral percutaneous low-to-low osteotomies were then performed. Tip exposure allowed precise placement of tip sutures, a columellar strut graft and a septal extension graft using septal cartilage. Alar rim grafts fashioned from the remaining conchal cartilage were inserted to correct the external valve collapse. The internal scar tissue was excised and adjacent mucosa rearranged to improve the nasal airway. Finally, the nasal base was narrowed by excision of small wedges from both nostrils. Comparison of the preoperative (FIGURE 2 LEFT) and 12-month postoperative (FIGURE 2 RIGHT) views demonstrates the correction of the nasal dorsum with creation of pleasing dorsal aesthetic lines, derotation of the nasal tip, resolution of the alar collapse, and narrowing of the nasal base.

\section{Case 2}

A 22-year old woman complained of a deviated nose, dorsal hump, asymmetric bifid tip and subtle breathing difficulties. The dorsal hump was addressed through the closed approach. Then, the open part of the approach allowed repositioning of the septum to the midline and fixation to the nasal spine caudally. Percutaneous low-to-low osteotomies were followed by placement of a columellar strut graft to stabilize both the tip and septum. Finally, the tip was refined with precise transdomal suturing under direct vision. Comparison of the 

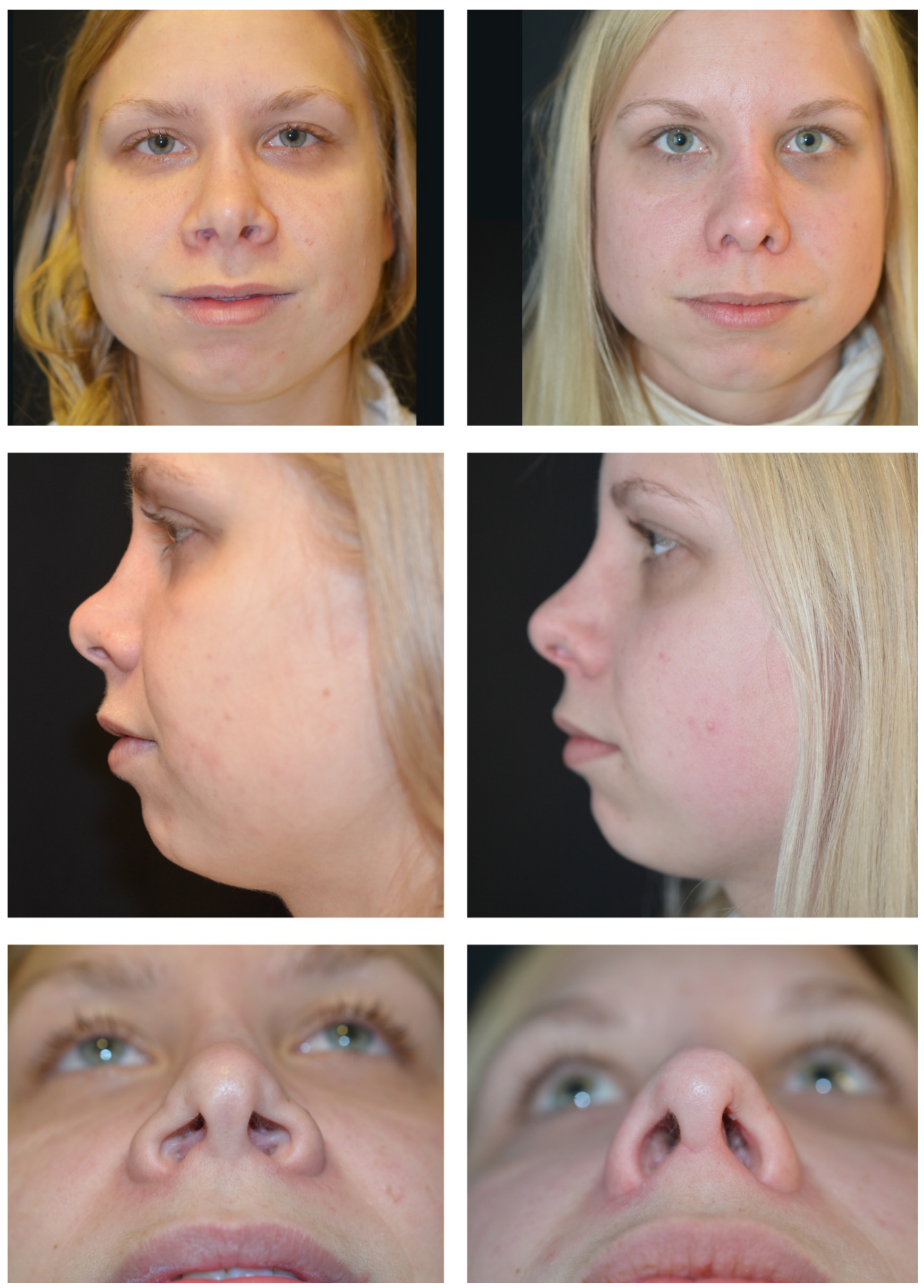

FIGURE 2: Preoperative and postoperative views of the patient in case 1.

preoperative (FIGURE 3 LEFT) and 12-month postoperative (FIGURE 3 RIGHT) views demonstrate correction of the nasal deviation with establishment of pleasing dorsal aesthetic lines, and improved nasal tip projection/ rotation.

\section{Case 3}

A 29-year old man complained of a crooked nose. Preoperative analysis (FIGURE
4 LEFT) showed a long nose with distorted dorsal aesthetic lines, a wide tip with poor support, a large infratip lobule and an acute labiocolumellar angle. Starting with the closed approach, dorsal hump reduction and cephalic trimming were performed. Percutaneous lowto-low osteotomies were completed prior to tip exposure. The nose was shortened with the help of spanning sutures, while the tip was supported with a columellar strut graft and covered with 

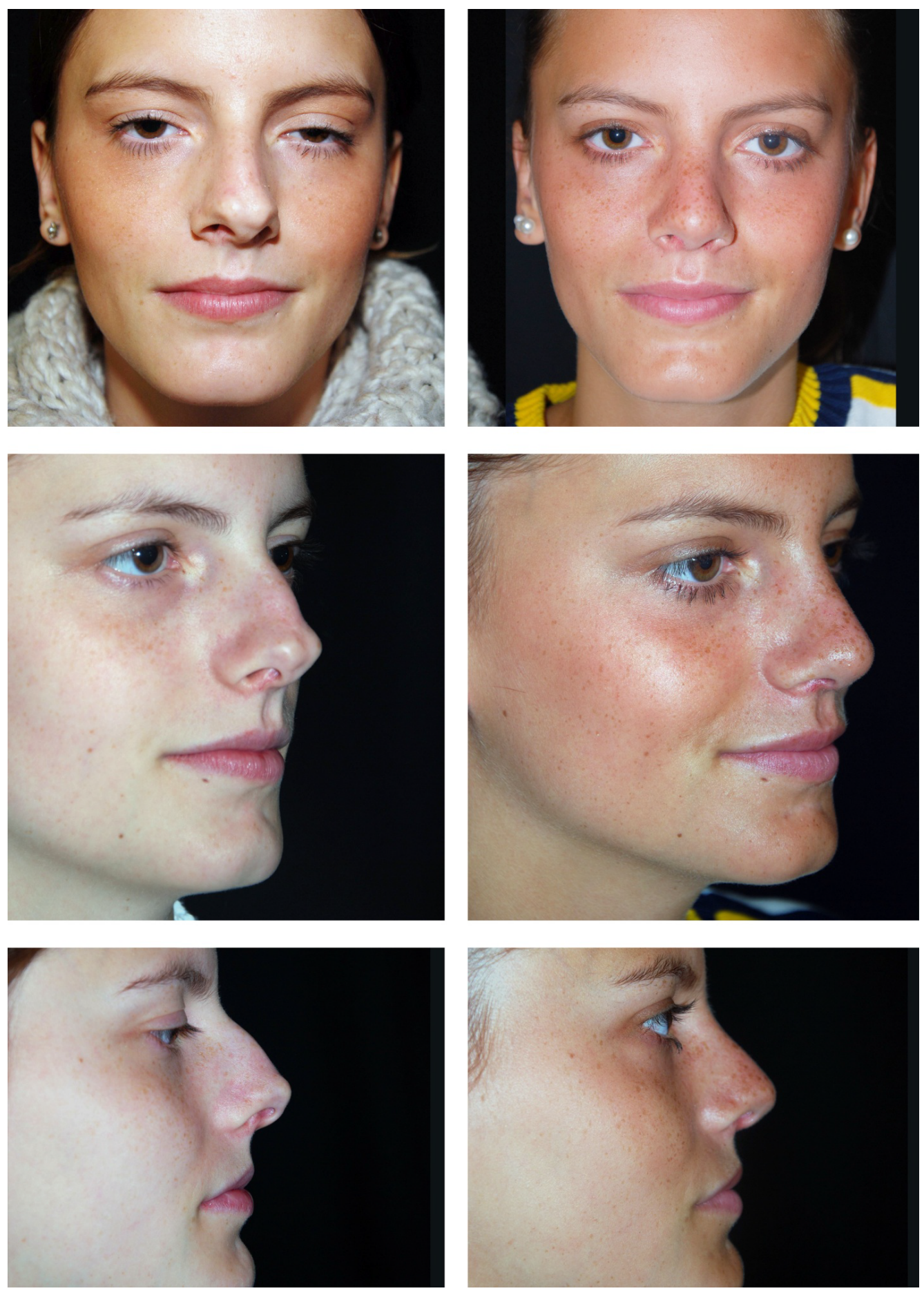

FIGURE 3: Preoperative and postoperative views of the patient in case 2.

a morcellated conchal shield graft. Comparison of the preoperative (FIGURE 4 LEFT) and 12-month postoperative (FIGURE 4 RIGHT) views demonstrate correction of the dorsal aesthetic lines and a balanced nasal profile.

\section{Discussion}

Rhinoplasty is undeniably a challenging and complicated surgical procedure with many steps and factors to consider. Small alterations lead to significant changes in the interplay of the tissue components. With so many variables to take into account, most surgeons become familiar with just one technical approach during their training [6-8] and feel comfortable to continue with that approach during their career. However, a specific approach is not a panacea for all problems encountered in rhinoplasty. Thus, although distinguished masters can attain and have published exceptional results with either one of 

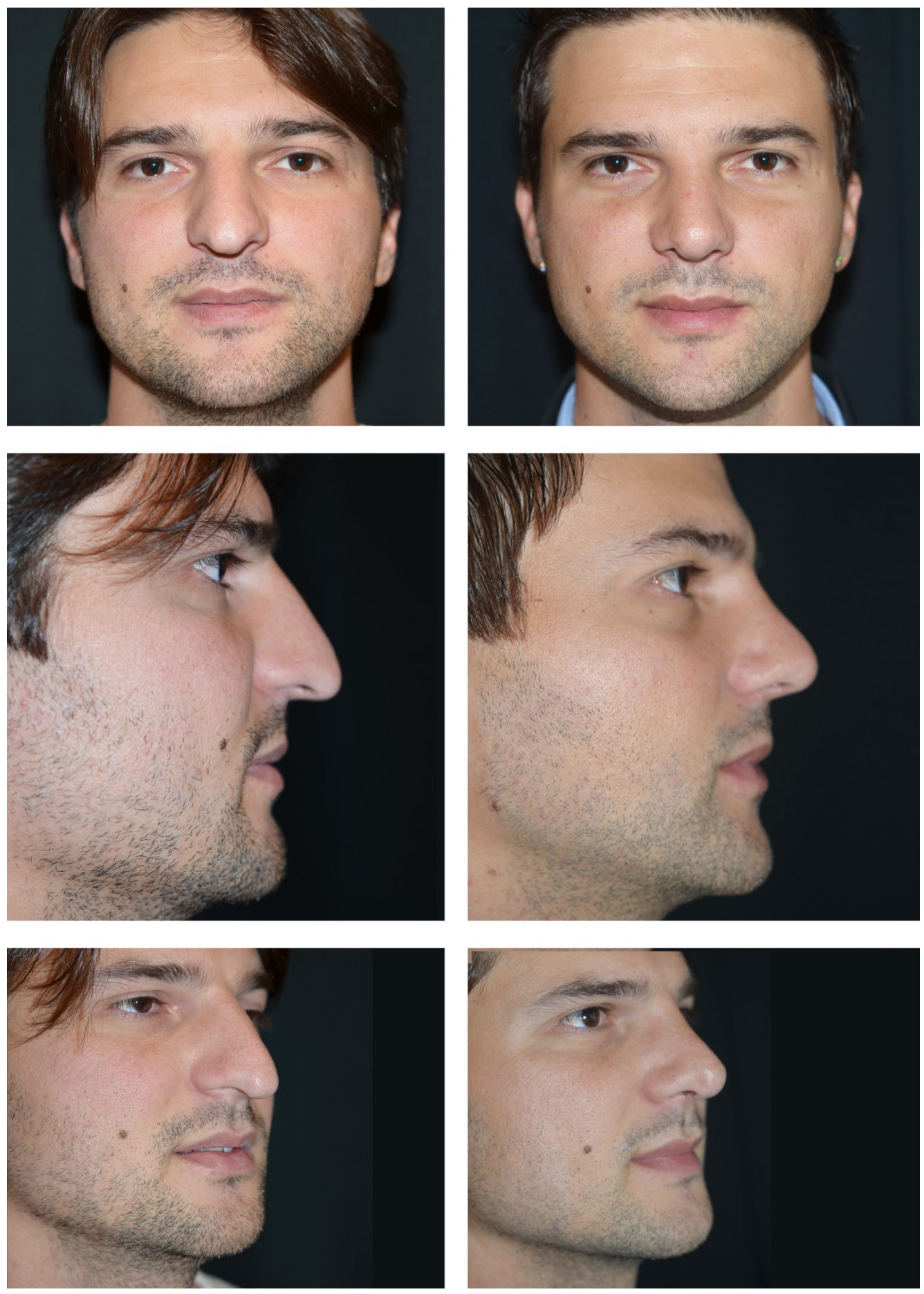

FIGURE 4: Preoperative and postoperative views of the patient in case 3.

the approaches, unselective application of this 'one-approach-fits-all' philosophy does not serve the best interest of each patient and surgeon. An antithesis to this philosophy is the graduated approach [8,9]. Each patient is individually assessed and allocated to the appropriate access based on the presenting anatomy, patient's desires and aesthetic goals. It allows the surgeon to have an open mind without being obsessed about avoiding the transcolumellar scar at any cost or dissecting widely no matter what. Instead, the emphasis is on achieving the preoperatively defined goals in the simplest, most efficient way while minimizing the extent of dissection and maintaining or fortifying structural support. The selected access is not a guarantee to successful outcome. Preoperative clinical assessment, detailed operative planning, selection of appropriate maneuvers and careful execution of them are as important, if not 
more so $[8,10]$. Nevertheless, for each chosen access the surgeon bares the consequences, both positive and negative. A central pillar of our graduated approach to rhinoplasty is tailoring the extent of dissection to provide optimum exposure for the selected maneuvers while avoiding unnecessary soft tissue manipulation. The planned maneuvers determine the access and amount of dissection. Noses with reasonable tip symmetry and definition requiring dorsal reduction or augmentation, cephalic trimming, and simple septoplasty are handled through a closed non-delivery approach with or without hemitransfixion incision. Simple revision cases that can be addressed through isolated maneuvers and camouflage grafts are also included in this category $[2,11]$. Asymmetric, broad, bifid, overor under-projected tip and significantly deviated septum are deformities compelling the surgeon to obtain greater exposure either through an extended closed tip delivery or a traditional open approach. Both require extensive dissection. The modified closed-open approach provides adequate exposure for accurate anatomic diagnosis and correction without wide dissection. Dorsal modification is still undertaken through a limited intracartilaginous incision and dissection. However, the tip is unroofed through a transcolumellar/limited marginal incision. The full scope of tip shaping and positioning maneuvers as well as complex septoplasty can be accomplished easily and reliably. This is true for complex revision rhinoplasty, too. Finally, the traditional open approach is reserved for patients requiring extracorporeal septoplasty, and for those being at risk of developing complex midvault deformities. The graduated and integrative approach aims at striking a balance between limited dissection, adequate exposure and effortless performance of the intended maneuvers by integrating both approaches.

Daniel has applied the 'closed-open approach' to access the dorsum and septum through an intercartilaginous incision and the tip through a traditional transcolumellar/ infracartilaginous incision. We have modified the approach by accessing the dorsum through an intracartilaginous incision instead and avoiding a transfixion incision to maintain an intact mucosal bridge at the internal valve area. In addition, the transcolumellar incision is extended only as a limited marginal incision just past the soft triangle. Having applied this technique in 482 complex primary and secondary rhinoplasties, over the past 10 years, we have noticed that there tends to be a decreased postoperative edema and scar formation when compared to open or extended closed approaches. Some surgeons do not regard postoperative edema an important complication in rhinoplasty. Yet, it is certainly more than a nuisance for the patient and can last up to a year or even longer in certain cases [12,13] Edema, fibrosis and scarring endanger the final aesthetic result by obscuring definition, even if the underlying framework is perfectly shaped. Male patients, those with thick skin, and revision cases, are at increased risk for postoperative edema and scarring [10,11,14-16]. Repeated postoperative visits for reassurance or steroid injections, lowers patient satisfaction and adds to surgeon's frustration. It is possible that the protracted edema, in most cases, is more related to the extent and plane of dissection than to the frequently blamed columellar incision [17]. The traditional open approach may predispose to dissection beyond what is absolutely necessary [2]. Unless the midline SMAS/Pitanguy ligament is preserved, the extended closed delivery approach can also cause significant edema due to the extensive and inherent nature of the dissection [18]. Through limited incisions and dissection, and by keeping mucosal bridges and a broad base of central and lateral alar margin undisturbed, the modified closed-open approach may partially avoid this complication. The often decreased operative time, compared to both open and closed delivery approaches, is an additional bonus. A drawback of the close-open approach, shared with the open, is the columellar scar. Poorly planned, cut or reapproximated columellar incisions may lead to depressed and notched scars that may not be obvious in the front view, but are easily visible on profile $[10,11]$. Among open rhinoplasty patients presenting for revision, 9 to $25 \%$ found the scar objectionable $[19,20]$. Nevertheless, properly closed by reppaproximation of SMAS to relieve skin tension and careful repositioning of the lateral edges, the scar can and should be imperceptible [21,22].

Alternative approaches to the traditional inter- or infracartilaginous delivery techniques have been described. They seek to provide wide exposure of nasal tip framework without the transcolumellar incision. The intercartilaginous incision is omitted, while the infracartilaginous incision is extended either medially to the midcollumella/nasal floor [19,23-25] or laterally, past the lateral crus towards the piriform aperture $[26,27]$ sometimes combined with alar base excisions [28]. Even though they do provide exposure of the alar cartilages some more than others, this is at the expense of more extensive dissection and they all require distortion of the alar cartilages during delivery through one nostril.

\section{Conclusion}


By incorporating the advantages of both open and closed approaches, and circumventing their limitations, the modified closed-open approach is a viable third option for complex cases. This approach should be considered as a hybrid approach bridging the gap between the two well established ones and is best utilized as a part of a graduated and integrative approach to rhinoplasty that integrates both open and closed techniques. 


\section{REFERENCES}

Daniel RK. Rhinoplasty: The First 100. In: Daniel RK, (editor). Rhinoplasty. 1st edn. Boston: Little Brown, 321380 (1993).

Tebbetts JB. Open and closed rhinoplasty (minus the "versus"): analyzing processes. Aesthet Surg J 26,456-459 (2006).

Perkins SW. The evolution of the combined use of endonasal and external columellar approaches to rhinoplasty. Facial Plast Surg Clin North Am 12,35-50 (2004).

Alexander RW. Fundamental terms, considerations, and approaches in rhinoplasty. Atlas Oral Maxillofac Surg Clin North Am 3,15-25(1995).

Toriumi DM, Mueller RA, Grosch T, et al. Vascular anatomy of the nose and the external rhinoplasty approach. Arch Otolaryngol Head Neck Surg 122(1),24-34 (1996).

Dayan SH. Evolving techniques in rhinoplasty. Facial Plast Surg 23,62-69 (2007).

Adamson PA, Galli SK. Rhinoplasty approaches: current state of the art. Arch Facial Plast Surg 7,32-37 (2005).

Simons RL, Grunebaum LD. The Endonasal Approach to Rhinoplasty. Ed. Rhinology and Facial Plastic Surgery. 1st edn. Berlin: SpringerVerlag, 713-721 (2009).

Cafferty A, Becker DG. Open and Closed Rhinoplasty. Clin Plast Surg 43,17-27 (2016).

Constantian MB. Constantian's Approach. In: Gunter JP, Rohrich RJ, Adams WP Jr, (editors). Dallas Rhinoplasty: Nasal Surgery by the Masters. 2nd edn St.
Louis: Quality Medical, 1465-1502 (2007).

Sheen JH. Closed versus open rhinoplasty-and the debate goes on. Plast Reconstr Surg 99,859-862 (1997).

Rohrich RJ, Ahmad J. Open technique rhinoplasty. In: Warren RJ, Nelligan PC, (editors). Plastic Surgery: Vloume 2: Aesthetic Surgery. 3rd edn. London: Saunders Elsevier 386-412 (2013).

Gunter JP. Gunter's Approach. In: Gunter JP, Rohrich RJ, Adams WP Jr, (editors). Dallas Rhinoplasty: Nasal Surgery by the Masters. 2nd edn. St. Louis: Quality Medical 1329-1354 (2007).

Rohrich RJ, Janis JE, Kenkel JM. Male rhinoplasty. Plast Reconstr Surg 112,1071-1185 (2003).

Gruber RP. Gruber's Approach. In: Gunter JP, Rohrich RJ, Adams WP Jr, (editors). Dallas Rhinoplasty: Nasal Surgery by the Masters. 2nd edn. St. Louis: Quality Medical 1439-1464 (2007).

Guerrerosantos J. Open rhinoplasty without skin-columella incision. Plast Reconstr Surg 85,955-960 (1990).

Adamson PA, Constantinides M, Kim AJ, et al. Rhinoplasty: panel discussion. Facial Plast Surg Clin North Am 22,25-55 (2014).

Cakir B, Oreroglu AR, Dogan T, et al. A complete subperichondrial dissection technique for rhinoplasty with management of the nasal ligaments. Aesthet Surg J 32,564-574 (2012).

Constantian MB. Differing characteristics in 100 consecutive secondary rhinoplasty patients following closed versus open surgical approaches. Plast Reconstr Surg 109,2097-2111 (2002).
Daniel RK. Secondary rhinoplasty following open rhinoplasty. Plast Reconstr Surg 96,1539-1546 (1995).

Cakir B. The Superficial SMAS. In: Cakir B (editor). Aesthetic Septorhinoplasty. 1 st edn. Cham: Springer International 290-291 (2016).

Rohrich RJ, Lee MR. External approach for secondary rhinoplasty: advances over the past 25 years. Plast Reconstr Surg 131,404-416 (2013).

Cárdenas-Camarena L, Guerrero MT. Improving nasal tip projection and definition using interdomal sutures and open approach without transcolumellar incision. Aesthetic Plast Surg 26,161-166 (2002).

Sevin A, Sevin K, Erdogan B, et al. Open rhinoplasty without transcolumellar incision. Ann Plast Surg 57,252-254 (2006).

Ors S, Ozkose M, Ors S. Comparison of Various Rhinoplasty Techniques and Long-Term Results. Aesthetic Plast Surg 39,465-473 (2015).

Holmström H, Luzi F. Open rhinoplasty without transcolumellar incision. Plast Reconstr Surg 97,321-326 (1996).

Kim PC, Park DH. External Marginal Approach Rhinoplasty (EMA Rhinoplasty). In: Shiffman MA, Di Giuseppe A, (editors). Advanced Aesthetic Rhinoplasty: Art, Science, and New Clinical Technique. 1st edn. Berlin: Springer 241-294 (2013).

Bravo FG, Schwarze HP. Closedopen rhinoplasty with extended lip dissection: a new concept and classification of rhinoplasty. Plast Reconstr Surg 122,944-950 (2008). 